\title{
Zur operativen Therapie post- operativer therapieresistenter Lymphozelen nach Eingriffen wegen einer Rezidivvarikose
}

\author{
P.-M. Baier; Z. T. Misczcak
}

Venen-Clinic Bad Neuenahr, Chirurgische Abteilung

\begin{abstract}
Schlüsselwörter
Lymphatische Komplikationen, Rezidivvarikose, postoperative Lymphozelen, chirurgische Therapie
\end{abstract}

\section{Zusammenfassung}

Es werden die Kasuistiken von zwei Patienten dargestellt, die wegen einer ausgeprägten Rezidivvarikose operiert werden mussten. Im postoperativen Verlauf entwickelten sich groBe und klinisch relevante Lymphozelen im Bereich der Innenseite des Ober- beziehungsweise Unterschenkels. Trotz intensiver konservativer Therapie kam es zur Progredienz. Aufgrund der ausgeprägten klinischen und sonographischen Befunde wurde die Indikation zur operativen Zystenresektion gestellt. Der anschließende postoperative Verlauf gestaltete sich komplikationslos und es kam zur vollständigen Restitution. Prinzipiell ist bei Operationen wegen einer Rezidivvarikose mit einem doppelt so hohem Risiko an postoperativen lymphatischen Komplikationen zu rechnen.

\section{Keywords}

Lymphatic complications, recurrent varicosis, postoperative lymphocysts, surgical therapy

\section{Summary}

The case reports are presented by two patients who had to undergo surgery for severe recurrent varicosis. In the postoperative course, large and clinically relevant lymphocysts developed. Despite intensive conservative therapy, it progressed. Due to the pronounced findings, the indication for operative cyst resection was asked. The subsequent postoperative course was uncomplicated and there was complete restitution. In principle, operations with recurrent varicosis are expected to be twice as likely to cause postoperative lymphatic complications.

For surgical treatment of postoperative lymphocels after surgery for recurrent varicosis Phlebologie 2018; 47: 127-131 https://doi.org/10.12687/phleb2403-3-2018 Eingereicht: 10. November 2017 Angenommen: 31. Januar 2018

English version available at: www.thieme.de/phlebo
Postoperative Lymphozelen (Synonym: Pseudo-Lymphzyste) nach Varizenoperationen wegen einer Rezidivvarikose sind recht häufig und zumeist harmlos, und oft sind sie nur eine lästige und unangenehme
Begleiterscheinung im unmittelbaren postoperativen Verlauf $(1,2,5,7,11,12)$. In der Vielzahl der Fälle treten sie nur vorübergehend auf und werden allein schon durch das Tragen von Kompressionsstrümpfen behandelt, oder aber sie kommen durch einfache therapeutische konservative Maßnahmen, wie etwa lokale Druckverbände, zur vollständigen Abheilung. Werden sie dagegen sehr groß oder entwickeln sich in Arealen mit einem großem Gefährdungspotential, dann sind songraphisch gestützte Zystenpunktionen mitunter erforderlich.

In Einzelfällen können sie aber den postoperativen Verlauf dahingehend verkomplizieren, dass nach Ausschöpfen aller konservativen Therapieoptionen ein operativer Zweiteingriff notwendig wird.

Wir möchten von zwei Fällen in unserem Krankengut der letzten beiden Jahre berichten, wo wir gezwungen waren, eine operative Zystenresektion im Rahmen eines Zweiteingriffs vorzunehmen.

\section{Fallbericht 1}

Bei dem 57-jährigem Patienten mit einem BMI von 36 erfolgte wegen eines beiderseitigen Mehrfachrezidivs mit inguinalen Varizenkonvoluten und Insuffizienz der V. saphena accessoria lateralis (VSAL) bei Dermatolipofasziosklerose (C 5 der CEAP Klassifikation) die beiderseitige Leistenrevision mit Ausschaltung der pathologischen Leistenrefluxe und Entfernung der Varizenkonvolute sowie proximaler Ligatur der VSAL. In gleicher Sitzung wurden die Seitenastvarikosen entfernt, phlebitischphlebosklerotischen Varizenareale und Fettgewebsnekrosen im Unterschenkelbereich exzidiert und die insuffizienten Perforansvenen transfaszial ligiert. Der Eingriff wurde in Laryngealmaskennarkose ( LM ) ausgeführt; die mittlere Operationszeit (Schnitt-Naht-Zeit) betrug $125 \mathrm{~min}$. Die Thromboembolieprophylaxe erfolgte 

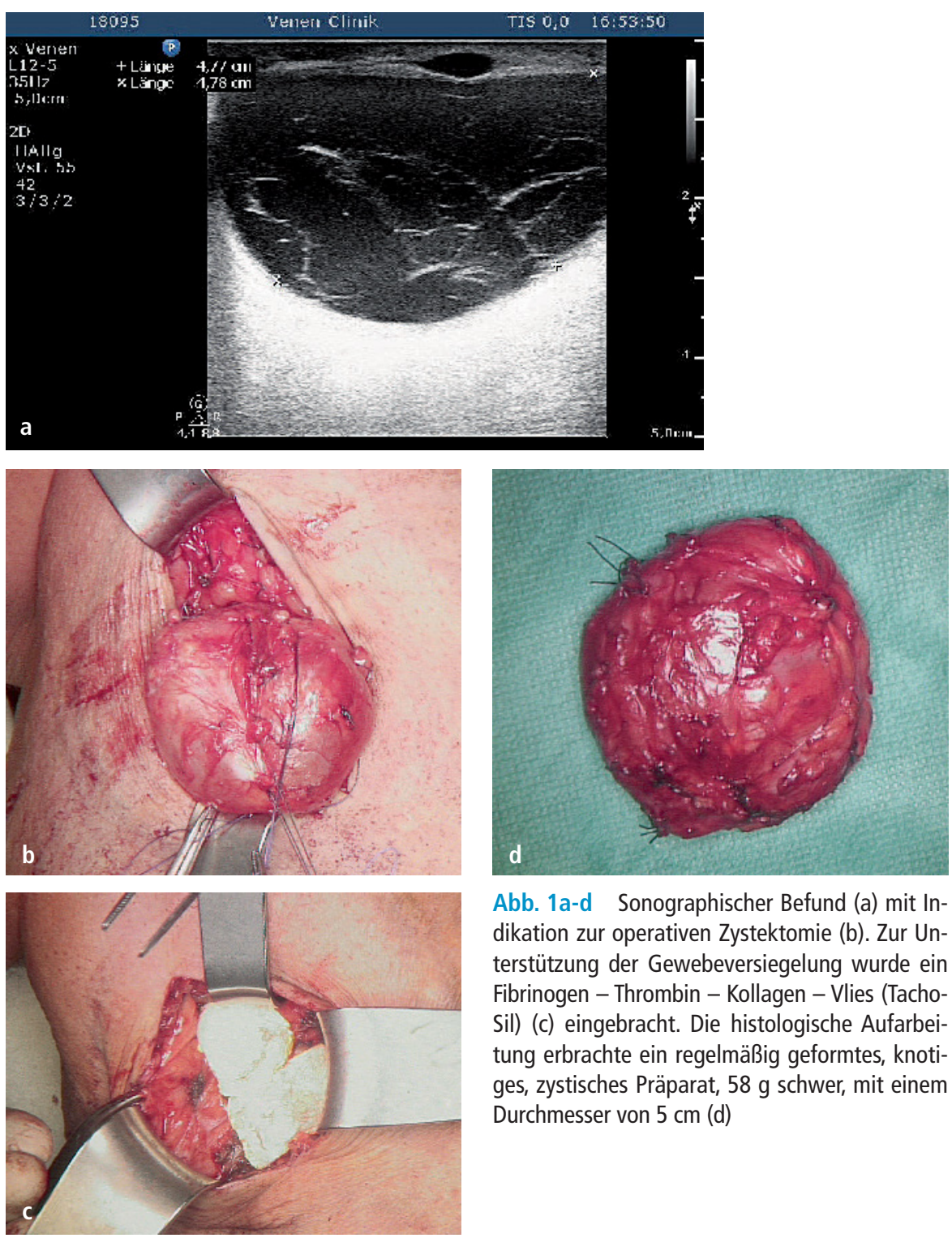

Abb. 1a-d Sonographischer Befund (a) mit Indikation zur operativen Zystektomie (b). Zur Unterstützung der Gewebeversiegelung wurde ein Fibrinogen - Thrombin - Kollagen - Vlies (TachoSil) (c) eingebracht. Die histologische Aufarbeitung erbrachte ein regelmäßig geformtes, knotiges, zystisches Präparat, $58 \mathrm{~g}$ schwer, mit einem Durchmesser von $5 \mathrm{~cm}$ (d)

mit Fondaparinux bis zum 14. postoperativen Tag. Der Patient wurde der ASA (American Society of Anaesthesia) II zugeordnet. Es lag ferner ein chronisches primäres Lymphödem I. Grades beiderseits vor. Der unmittelbare postoperative Verlauf gestaltete sich zunächst komplikationslos und der Patient wurde mit reizlosen Wundverhältnissen und flachgestrickter Kompressionsbestrumpfung der Klasse II (Größe A-G) am 2. postoperativen Tag entlassen. Bei der ambulanten Wiedervorstellung am 10. postoperativen Tag fand sich klinisch und sonographisch eine Lymphozele im Bereich der linken proximalem Unterschenkelinnenseite, die zusätzlich zu den
Normbereich. Es wurden weiterhin, zusätzlich zur flachgestrickten Kompressionsbestrumpfung, lokale Druckverbände angelegt und zweimal wöchentlich manuelle Lymphdrainagen durchgeführt.

Drei Wochen später entwickelte sich bei dem Patienten ein Erysipel im Bereich des linken distalen Unterschenkels mit Fieber und Schüttelfrost, Schwellung und Erythem, Leukozytose und CRP-Anstieg auf $59 \mathrm{mg} / \mathrm{l}$ (Referenzbereich 0,1-5,0). Es erfolgten unter stationären Bedingungen die Lagerung des Beines auf einer Braun $\searrow$ schen Schiene, lokal antiseptische Verbände mit Povidon-Iod-Lösung, intravenöse Antibiose mit Aminopenicillin, Thromboembolieprophylaxe mit Fondaparinux und analgetisch-antiphlogistische Therapie. Darunter kam es zur vollständigen Rückbildung der inflammatorischen Gewebeveränderung und der Patient wurde fieberfrei mit normalen CRP-Werten entlassen. Farbduplexsonographisch fand sich unverändert eine ca. $4 \times 4 \mathrm{~cm}$ große, reizlose Lymphozele im Bereich der proximalen Unterschenkelinnenseite und die konservative Therapie mit Tragen von flachgestrickten Kompressionsstrümpfen der Klasse II wurde fortgesetzt. Trotzdem kam es nicht zu einer Regredienz der Lymphozele.

Sechs Monate nach dem Primäreingriff stellte sich der Patient erneut wegen Schmerzhaftigkeit und Schwellungszuständen im Bereich des linken Unterschenkels vor. Sonographisch fand sich unverändert eine etwa $4 \times 4 \mathrm{~cm}$ große, zystische und gekammerte Raumforderung im Bereich der linken proximalen Unterschenkelinnenseite. Aufgrund der klinischen Symptomatik und des sonographischen Befundes wurde die Indikation zur operativen Zystektomie gestellt. Der Eingriff erfolgte stationär in LM-Narkose, die Operationszeit betrug 95 min. Intraoperativ fand sich eine $5 \times 6 \mathrm{~cm}$ große Lymphozele, die schrittweise freigelegt wurde und in toto entfernt werden konnte. Das speisende Lymphgefäß im proximalen Anteil konnte separiert und mit einer Umstechungsligatur versorgt werden. Zur Unterstützung der Gewebeversiegelung wurde ein FibrinogenThrombin-Kollagen-Vlies (TachoSil) neben einer lokalen Redon-Drainage in die ehemalige Zystenloge eingebracht. Der 
Wundverschluss erfolgte primär, und ein Kompressionsverband wurde angelegt. Bis zum 3. postoperativen Tag erfolgte eine i.v.Antibiose mit Aminopenicillin; die Thromboembolieprophylaxe wurde bis zum 14. postoperativen Tag mit Fondaparinux fortgesetzt.

Die histologische Aufarbeitung erbrachte ein regelmäßig geformtes, knotiges, zystisches Präparat, $58 \mathrm{~g}$ schwer, mit einem Durchmesser von $5 \mathrm{~cm}$. Immunhistochemisch wurde die klinische Diagnose einer Lymphozele bestätigt.

Der anschließende postoperative Verlauf gestaltete sich komplikationslos. Die sonographische Kontrolle nach 6 Wochen zeigte bis auf diskrete narbige Residuen im Operationsgebiet einen unauffälligen $\mathrm{Be}$ fund ( $>$ Abb. 1a bis 1d).

\section{Fallbericht 2}

Die 76-jährige ASA-III-Patientin mit einem BMI von 26 und dauerantikoaguliert mit Phenprocoumon wegen Vorhofflimmern musste wegen einer beiderseitigen Rezidivvarikose mit pathologischem Leistenreflux infolge Insuffizienz der VSAL und Seitenastvarikosen und Varizenkonvoluten im Ober- und Unterschenkelbereich bei C 4 der CEAP-Klassifikation operiert werden. Dabei wurden in LM-Narkose eine beiderseitige Leistenrevision mit Abtragung der inguinalen Varizenkonvolute, proximaler Ligatur der VSAL, minichirurgischer Phlebextraktion der Seitenastvarikosen und Varizenkonvolute vorgenommen. Die Operationszeit betrug $95 \mathrm{~min}$. Bis zum 2. postoperativen Tag erfolgte die Thromboembolieprophylaxe in gewichtsadaptierter Form mit niedermolekularem Heparin (Nadroparin 0,8 2 x täglich). Anschließend wurde die Patientin entsprechend dem Bridging-Schema wieder auf Phenprocoumon eingestellt. Der unmittelbare postoperative Verlauf gestaltete sich komplikationslos, und die Patientin wurde mit reizlosen Wundverhältnissen am 2 . postoperativen Tag mit rundgestrickten Kompressionsstrümpfen der Klasse II (Größe A-G) entlassen. Nach dem 10. postoperativen Tag bildete sich eine derbe, schmerzhafte Verhärtung im Bereich der Innenseite des distalen Oberschenkels he-
Abb. 2a-c

Sonographisch stellte sich konstant die therapieresistente Lymphozele mit einer GröBe von $2,5 \times 3,5 \mathrm{~cm}$ dar (a), Intraoperativ (b) konnte eine 3,5 $\mathrm{x}$ 3,0 große, abgekapselte Zyste im Bereich der linken Oberschenkelinnenseite vollständig exstirpiert werden (c).
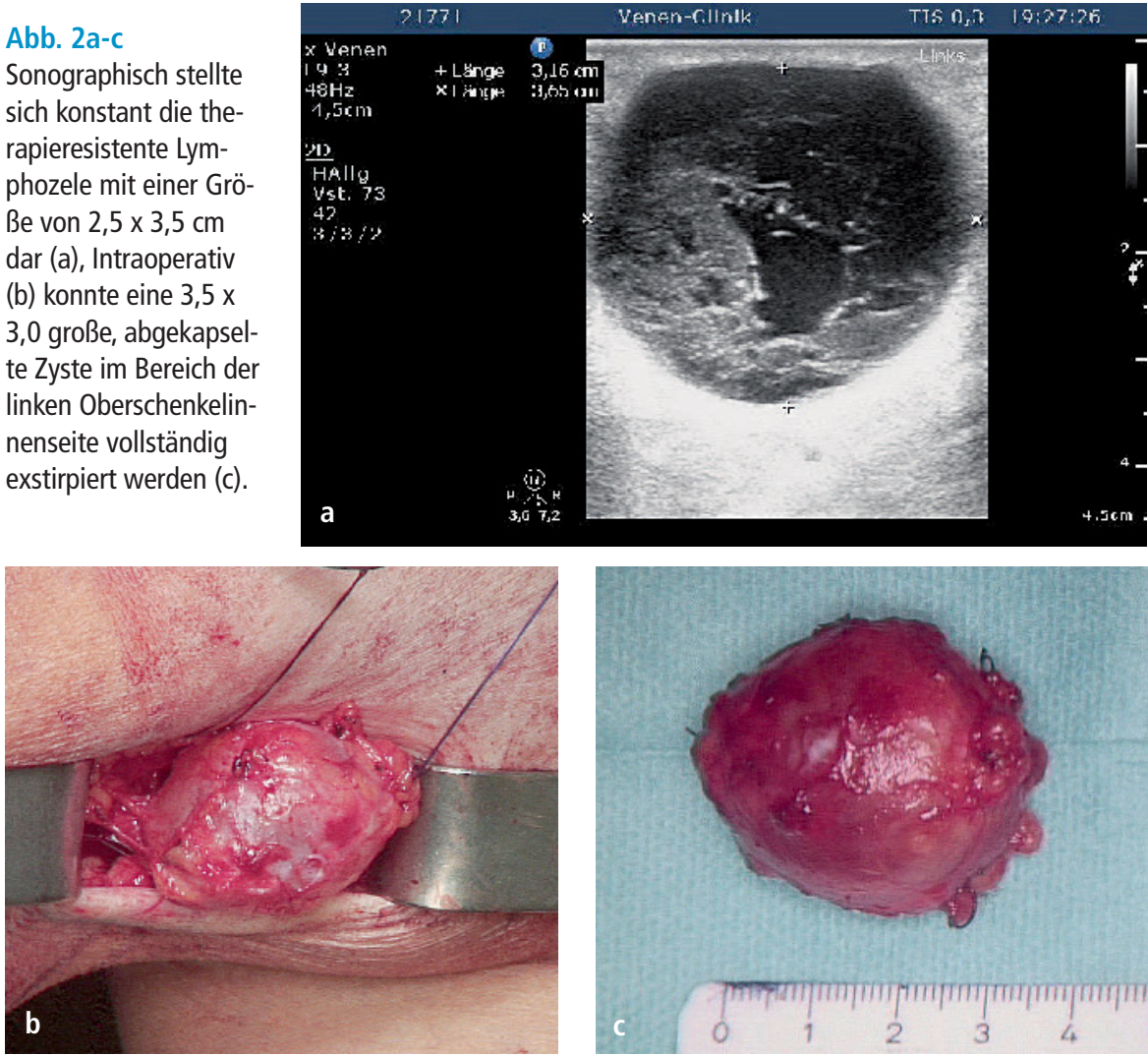

raus. Sonographisch fand sich eine 2,5 x 3,5 $\mathrm{cm}$ große, reizlose Lymphozele . Zunächst wurden zusätzlich zur Kompressionsbestrumpfung lokale Druckverbände angelegt und manuelle Lymphdrainagen verordnet. Nach vier Wochen stellte sich die Patientin erneut wegen anhaltender lokaler Schmerzen, verbunden mit Schwellungszuständen im Bereich des linken Oberschenkels, vor. Farbduplexsonographisch fand sich bei unauffälligem tiefen Beinvenensystem unverändert eine 2,5 x $3,5 \mathrm{~cm}$ große Lymphozele im Bereich der distalen Oberschenkelinnenseite. Aufgrund der klinischen Symptomatik wurden sonographisch gestützt 30 $\mathrm{ml}$ seröse Flüssigkeit abpunktiert und ein lokaler Druckverband angelegt. Dennoch kam es nach einer Woche zum Nachlaufen der Zyste mit entsprechender Klinik. Zunächst wurde mit der Patientin eine weitere konservative Vorgehensweise mit konsequentem Tragen von Kompressionsstrümpfen und zweimal wöchentlichen manuellen Lymphdrainagen vereinbart.

Vier Monate nach dem Primäreingriff fand sich unverändert die schmerzhafte, derbe Anschwellung medialseitig des lin- ken distalen Oberschenkels. Auch sonographisch stellte sich konstant die therapieresistente Lymphozele mit einer Größe von $2,5 \times 3,5 \mathrm{~cm}$ dar, wobei sich nun auch distal davon Lymphabflussstörungen mit vermehrten Lymphspalten entwickelten. Basierend auf den klinischen und sonographischen Befunden wurde die Indikation zur operativen Zystenresektion gestellt.

Die Operation wurde in LM-Narkose ausgeführt, die Operationszeit betrug 75 min. Intraoperativ konnte eine 3,5 x 3,0 große, abgekapselte Zyste im Bereich der linken Oberschenkelinnenseite vollständig exstirpiert werden. Das zuführende Lymphgefäß wurde im proximalen Anteil verifiziert und mit einer Umstechungsligatur versorgt. Neben einer lokalen RedonDrainage wurde ein Fibrinogen-Thrombin-Kollagen-Vlies (TachoSil) in die ehemalige Zystenloge platziert, um die Gewebeversiegelung zu beschleunigen. Es erfolgten ein primärer Wundverschluss und die Anlage eines Kompressionsverbandes. Am 2. postoperativen Tag wurde die Thromboembolieprophylaxe in gewichtsadaptierter Form (Nadroparin 0,8 2 x täglich) entspre- 
chend dem Bridging-Schema überlappend auf Phenprocoumon umgestellt.

Histologisch fand sich ein $36 \mathrm{~g}$ schweres, zystisches, $3,5 \times 3,5 \times 3.0 \mathrm{~cm}$ messendes, mit klarer Flüssigkeit gefülltes Exzidat mit glatter Innen- und Außenwand. Immunhistochemisch wurde das Vorliegen einer Lymphozele bestätigt.

Der weitere postoperative Verlauf gestaltete sich unauffällig und bei der Kontrolluntersuchung nach sechs Wochen war die Patientin beschwerdefrei. Sonographisch fanden sich nur noch einzelne narbige Residuen im Gebiet der ehemaligen Zystenloge ( $\triangleright$ Abb. 2a bis 2c ).

\section{Diskussion}

Die Varizenchirurgie gilt heute, in einer Zeit, in der sich alternative Therapien, allen voran die endovenösen Ablationsverfahren, zunehmend etabliert haben, als rasch erlernbar, schnell und einfach durchführbar, wenig aufwändig und mit nur wenigen postoperativen Risiken behaftet. Dem wird aber die tatsächliche klinische Praxis in keiner Weise gerecht. Denn bei allem technischen Fortschritt und allen Trends zur minimalinvasiven Vorgehensweise ist auch heute noch die Chirurgie der Varikosis sehr mühsam und zeitaufwändig und setzt große operative Erfahrungen voraus, vor allem dann, wenn ausgeprägte Befunde, Rezidivvarikosen, Komplikationen des Krampfaderleidens und eine progrediente chronisch-venöse Insuffizienz (CVI) vorliegen. Diese Patienten sind es dann auch, die mitunter postoperativ zu Komplikationen neigen und somit alle Beteiligten vor Probleme stellen können. Nicht die MajorKomplikationen stellen das eigentliche Problem dar, denn diese sind ja nur Einzelfälle, sondern die weniger essenziellen Minor-Komplikationen, allen voran die lymphatischen, sind für die Patienten unangenehm und lästig und erfordern manchmal einen erneuten chirurgischen Eingriff $(1,2$, $4,5,7,9,12,13)$.

Lymphatische Komplikationen in der Krampfaderchirurgie sind zwar häufig, jedoch spiegelt sich dieser Umstand nicht in der Literatur wider. Hier schwanken die lymphatischen Komplikationsraten zwischen 0,15 (11) und 6,2\% (3). Im Rahmen einer prospektiven Studie lagen unsere Quoten bei 4,5\%, wobei Patienten mit einer Rezidivvarikose doppelt so häufig lymphatische Komplikationen postoperativ aufwiesen als Patienten nach einer primären Varizenoperation (1).

Postoperative Lymphozelen treten häufig auf und entstehen dann, wenn der Lymphabstrom in seiner Kontinuität unterbrochen wird und so die Lymphe aus einem verletzten Gefäß nicht abfließen kann und sich eine Pseudokapsel ausbildet. Hierbei besitzt der Verlauf des ventromedialen Lymphbündels im Bereich der Innenseite des distalen Unterschenkels ein sehr hohes Gefährdungspotential, welches signifikant ansteigt, sobald eine fortgeschrittene CVI, ein postthrombotisches Syndrom oder ausgeprägte Rezidivvarikosen mit Adhäsionen bereits präoperativ bestanden haben $(1,4$, 5, 11-13).

Im Großteil der Fälle führt das Ausschöpfen der konservativen Maßnahmen lokale Druckverbände in Kombination mit manueller Lymphdrainage - zum Sistieren der Lymphsekretion durch Verklebung der geschädigten und sezernierenden Lymphgefäße und damit zu einer vollständigen Restitution. Eine prophylaktische antibiotische Abschirmung ist nicht erforderlich. Sonographisch gestützte Zystenpunktionen unter aseptischen Bedingungen mit lokalen Druckverbänden sollten, wegen der Möglichkeit einer Keimverschleppung, nur großen, mechanisch irritierenden Zysten vorbehalten sein $(5,11)$.

In Einzelfällen scheint auch die Installation von Polidocanolschaum bei persistierenden, therapieresistenten Lymphozelen erfolgreich zu sein (10). Daneben wird die Injektion des Antibiotikums Doxycyclin in therapierefraktären Fällen in der Literatur positiv bewertet. Hierbei soll eine lokale inflammatorische Reaktion induziert werden, die zu einer Extravasation von Fibrin führt, wodurch die Zystenwände konsekutiv verkleben und es damit zum Versiegen der Lymphsekretion kommt $(6,8)$.

Greifen die konventionellen konservativen Therapien nicht, dann sind bei persistierenden oder progredienten und gleichzeitig klinisch relevanten Lymphozelen mitunter chirurgische Maßnahmen angezeigt.
Wir sind, wie Hach (5), der Meinung, dass sekundäre Eingriffe wegen therapierefraktären Lymphozelen prinzipiell unter stationären Bedingungen stattfinden sollten. Anzustreben ist hierbei immer die vollständige Resektion der Pseudozyste mit Darstellung und Versorgung des sezernierenden Lymphgefäßes, entweder elektrochirurgisch oder mittels Umstechungsligatur. Wir legen neben einer lokalen RedonDrainage ein Fibrinogen-Thrombin-Kollagen-Vlies (TachoSil) in den Wundgrund der ehemaligen Zystenloge. Dabei wird sowohl das Fibrinogen als auch das Thrombin aktiviert, was zu einer Beschleunigung der lokalen Gewebeversiegelung führt. Ein primärer Wundverschluss wird immer angestrebt und die Anlage eines Kompressionsverbandes ist obligat.

\section{Schlussfolgerung}

Postoperative Lymphozelen nach Krampfaderoperationen sind häufig; sie sind überwiegend harmlos und bedürfen neben der Kompression zumeist keiner speziellen Therapie.

Erst wenn das Reservoir der konservativen Therapieoptionen vollständig ausgeschöpft ist, sollten bei therapieresistenten, persistierenden Lymphozelen auch operative Revisionseingriffe zum Einsatz kommen.

Bei Rezidiveingriffen ist mit einem mehr als doppelt so hohem Risiko an postoperativen lymphatischen Komplikationen zu rechnen. Diese Tatsache sollte zwingend bei der Patientenaufklärung berücksichtigt werden.

Gerade bei Revisionsoperationen ist eine schonende, atraumatische Operationstechnik mit elektrochirurgischer Blutstillung unabdingbar. Für den Operateur setzt dies neben einer großen Erfahrung unbedingt anatomisch-topographische Kenntnisse des Lymphgefäßsystems voraus.

In den besonders gefährdeten Regionen, ventralseitig im Bereich des distalen Unterschenkel und in der medialen Knieregion, sollte bei Rezidivvarikosen auf die minichirirgische Haken-Phlebextraktion verzichtet werden. Lymphschonender ist hier die Darstellung und Entfernung der Varizen 
unter Sicht über kleine, longitudinale Hautinzisionen.

Deshalb sollten die operativen Zugangswege wie auch die operativen Strategien und Techniken klinikintern standardisiert werden und sich in einer hauseigenen SOP (Standard Operating Procedure) widerspiegeln.

\section{Interessenkonflikt}

Die Autoren geben an, dass kein Interessenkonflikt besteht.

\section{Ethische Richtlinien}

Die für dieses Manuskript erhobenen Daten stimmen mit den nationalen Gesetzen und der aktuellen Helsinki-Deklaration überein, eine Einverständniserklärung des Patienten lag vor.

\section{Literatur}

1. Baier PM, König N, Miszczak ZT, Stark HJ. Lymphatische Komplikationen nach Varizenoperationen bei fortgeschrittener chronisch-venöser Insuffizienz. Phlebologie 2008; 37: 227-282.

2. Balzer K. Komplikationen bei Varizenoperationen. Zentralbl Chir 2001; 126: 537-542.

3. Disselhoff BC, der Kinderen DJ, Moll FL. Is there a risk for lymphatic complications after endovenous Laser treatment versus cryostripping of the great saphenous vein? A prospective study. Phlebology 2008; 23: 4-10.

4. Fischer R, Früh G. Die Varizenoperation beim Vorliegen eines Lymphödems - wann ist sie sinnvoll? Phlebologie 1998; 27: 181-184.

5. Hach W. Venenchirurgie. Stuttgart: Schattauer 2006; S. 155.

6. Hackert T, Werner J, Loos M, Büchler MW, Weitz, J. Successful doxycycline treatment of lymphatic fistula: Report of five cases and review of the literature. Langenbecks Arch Surg 2006; 391: 435-438.

7. Hofer T. Komplikationen nach varizenchirurgischen Eingriffen. Phlebologie 2001; 30: 26-30.
8. Juntermanns B, Cyrek AE, Bernheim J, Hoffmann JN. Manegment von Lymphfisteln in der Leistenregion aus chirurgischer Sicht. Chirurg 2017; 88: $582-586$.

9. Kröger K, Lulay G, Miller A. Lymphödeme in der Gefäßchirurgie. Gefäßchirurgie 2016; 21: 572-579.

10. Moritz RKC, Reich-Schupke S, Altmeyer P, Stücker M. Polidocanol - Schaumsklerosierungstherapie von persistierenden postoperativen Seromen nach Varizenoperationen. Vasomed 2013; 25: 199-201.

11. Nüllen $H$, Noppeney $T$. Lymphgefäßläsionen bei der Varizenbehandlung. In: Noppeney T, Nüllen H. Diagnostik und Therapie der Varikose. Springer Heidelberg 2010; S 386-388.

12. Öhlbauer M, Mildner A, Brenner E, Hilbe G. Lymphzysten und Fisteln nach Varizenoperationen. Lymphologie in Forschung und Praxis 2000; 4: 11-13.

13. Ouvry PA, Guenneguez H, Ouvry PAG. Les complications lymphatiques de la chirugie des varices. Phlebologie 1993; 46: 563-568. 